\title{
Evaluating Smartphone Accuracy for RSSI Measurements
}

\author{
Yanis Boussad, M. Naoufal Mahfoudi, Arnaud Legout, Leonardo Lizzi, Fabien Ferrero \\ Walid Dabbous
}

\begin{abstract}
-
Smartphones are today affordable devices, capable of embedding a large variety of sensors such as magnetometers or orientation sensors, but also the hardware needed to connect them to most wireless communication technologies such as Wi-Fi, Bluetooth, or cellular networks. Therefore, they are handy devices able to perform Received Signal Strength Indicator (RSSI) measurements for a wide variety of applications such as cellular coverage maps, indoor localization, or proximity tracking. However, to the best of our knowledge, the accuracy of such measurements has never been rigorously assessed. The goal of this paper is to assess the accuracy of the RSSI measurements made with a Commercial Off-The-Shelf (COTS) smartphone in a variety of conditions, and how possible inaccuracies can be corrected. We primarily focus on the LTE RSSI, but we also extend our results to the Bluetooth RSSI.

In this paper, we build a controlled experimental setup based on commodity hardware and on open-source software. We evaluate the granularity and limitations of the Android API that returns the RSSI. We explore how reliable the measurements in a controlled environment with a monopolarized antenna are. We show that the orientation of the smartphone, the position or orientation of the source, and the transmission power have a significant impact on the accuracy of the measurements. We introduce several correction techniques based on radiation matrix manipulations and on machine learning in order to improve measurement accuracy to less than $5 \mathrm{dBm}$ RMSE, as compared to a professional equipment.

We also explore the reliability of measurements made in an outdoor realistic environment. We show that whereas transmission diversity available in LTE base stations significantly improves the measured RSSI regardless of the smartphone orientation, the Bluetooth RSSI remains largely sensitive to the smartphone orientation.
\end{abstract}

Index Terms - RSSI, LTE, accuracy, Bluetooth, calibration, sensors, smartphone, SDR

\section{INTRODUCTION}

Smartphones are sophisticated devices with a lot of embedded sensors, but also with the support of several wireless technologies, such as Wi-Fi, Bluetooth, 2G, 3G, 4G, and now 5G. For this reason, they are used to make measurements of the Received Signal Strength Indicator (RSSI). Such measurements are important in multiple contexts. We discuss a few such important practical contexts in the following paragraphs. First, companies such as OpenSignal [1] or Tutela [2] make crowd-based cellular measurements which are sold to cellular operators in order to improve their network. Indeed, the RSSI as experienced

Y.Boussad. Université Côte d'Azur, Inria, France. e-mail: yanis.boussad@inria.fr

M. Naoufal Mahfoudi. University of California San Diego, US.

A. Legout and W. Dabbous are with the Université Côte d'Azur, Inria, France.

F. Ferrero and L. Lizzi are with the Université Côte d'Azur, CNRS, LEAT, France. by the consumers is key to evaluate the coverage of the cellular network and to decide where to place additional cells. Operators also use this information to have a better understanding about the competition. Second, large European projects, such as EMF-NET [3], Interphone [4], or GERoNiMO [5] explored the impact on human health of the exposure to electromagnetic fields. In particular, the Interphone study [4] is at the origin of the classification of radiofrequency EMF as carcinogenic $2 \mathrm{~B}$ by the World Health Organization [6]. However, all their studies faced the difficulty consisting in accurately quantifying the exposure of the population to radiofrequency electromagnetic fields. For this reason, the European project COMOS [7] developed an Android app called XMobisense [8] aimed to the measurement of the exposure of a cohort during an experiment. This app had a confidential usage and is no longer maintained. The ElectroSmart project [9] also developed an Android application to inform the general audience about their levels of exposure. This project is still active and has $200 \mathrm{k}$ monthly users. Third, indoor positioning is a significant problem. One of the approaches to address this problem is to use Bluetooth beacons. In that context, the accurate estimation of the RSSI is important in order to determine the proximity to the surrounding beacons [10]. Last but not least, proximity tracking is of growing interest in order to trace proximity contacts in the context of the COVID-19 pandemic. All these applications are based on the Bluetooth technology[11][12] and an accurate estimation of the Bluetooth RSSI is of crucial importance to infer proximity. Whereas, the accuracy of the RSSI measurements is a key point in all these contexts, this is still a topic difficult to approach and, to the best of our knowledge, there is no rigorous evaluation of this accuracy for COTS smartphones.

In this work, we evaluate the accuracy of an Android COTS smartphone when performing measurements of the RSSI emitted from a 4G (LTE) source, and we extend our results to a Bluetooth source. Our contributions are the following. i) We evaluate the granularity and limitations of the Android API that provides the RSSI. We show that not all methods to access the RSSI are equivalent. We can expect a $2 \mathrm{~dB}$ granularity and an update every second (at the most) for the measurements made. ii) We explore the accuracy of the RSSI measurements in a fully controlled environment with a mono-polarized antenna. We show that the accuracy of the measurements is extremely sensitive to the device orientation, source positioning and orientation, and also to the source Tx power. iii) We propose several correction techniques aimed to the improvement of the ac- 
curacy of the RSSI that rely on manipulations of radiation matrices and on machine learning. We show that we can significantly improve the accuracy and obtain a Root Mean Square Error (RMSE) lower than $5 \mathrm{dBm}$ as compared to a calibrated professional equipment. iv) We explore the accuracy of the RSSI measurements in an outdoor realistic environment. We show that transmission diversity available for the LTE base stations dramatically reduces the RSSI sensitivity to the device orientation. However, transmission diversity is not available for all wireless technologies. In particular, we show that the Bluetooth RSSI is still sensitive to the device orientation in a realistic environment v) We make available all the RSSI correction artifacts and measurement data to the industry and to the public in general, in addition to the precomputed calibration matrices for an easier reusability [13].

\section{Related work}

Previous works explored the possibility to perform measurements of the received power, but not for COTS devices with no additional dedicated hardware. Tan et al. proposed Snoopy [14], a spectrum analyzer that uses commodity Wi-Fi cards with frequency translators in order to sense a wide range of frequencies. The Wi-Fi card normally scans only at $2.4 \mathrm{GHz}$ and $5 \mathrm{GHz}$. To extend this range and to scan a wider spectrum, Snoopy uses an RF frequency translator that senses and translates the signals to adapt them to the frequency supported by the Wi-Fi card. This is not readily applicable to COTS smartphones since they do not expose RF connectors of their Wi-Fi cards. Another work that aimed at using a commodity smartphone as a spectrum analyzer is presented by Ana et al. [15]. They make use of a portable Software Defined Radio (RTL-SDR) dongle that senses a continuous spectrum range from $52 \mathrm{MHz}$ to $2200 \mathrm{MHz}$, which they connect to a smartphone through USB. The dongle is the spectrum analyzer. The smartphone only processes the data from the dongle. In contrast to the two aforementioned works that rely on external hardware, CrowdREM [16] relies only on smartphones for spectrum analysis. The authors used an open-source mobile phone (OpenMoko [17]) on which they installed a modified Linux system and replaced the whole baseband system by OsmocomBB [18], an open-source GSM baseband implementation. They showed that smartphone accuracy is within $3 \mathrm{dBm}$ while the device is still, however it is very sensitive to the orientation with respect to the source, a difference of up to $10 \mathrm{~dB}$ difference. Smartphones have also been used for network measurements and analysis. Vallina-Rodriguez et al. [19] developed a mobile application run on Android devices to monitor the network performance using low-level radio information. They used the Radio Interface Layer (RIL) to access radio message exchange between the Android OS and the baseband chip. Their solution works only on Android devices with Intel Infineon XGold chip, and requires root-privileges (software modification on the Android OS).

As opposed to the previous works, the solution we propose in this paper relies solely on an off-the-shelf smart-

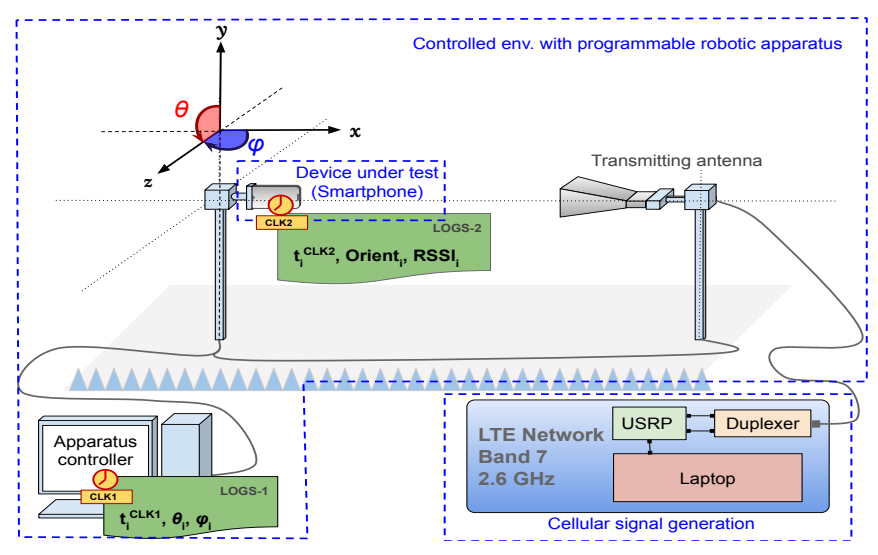

Fig. 1. The controlled experimental setup. SDR is used for signal generation and a two-axis positioning system is used to rotate the device-under-test in order to study the effect of orientation on the reception performance.

phone without any external hardware, hardware modification, or software modification (no rooting and no custom operating system required) on the smartphone. Moreover, we mitigate the inaccuracy of smartphones [16] with a correction technique that uses the Inertial Measurements Units (IMUs) of the mobile device in order to determine the correction power offset which has to be applied.

The impact of orientation on the RSSI and RSSIdependent applications for indoor positioning and localization has been further discussed in the literature. Li et al. [20] showed that RSSI from COTS RFID tags can vary by more than $15 \mathrm{~dB}$ across different orientations. Pasku et al. [21] investigated the effect of antenna directivity and receiver orientation on the RSSI in the $2.4 \mathrm{GHz}$ band for RF ranging applications. They showed that the RSSI from a mobile system containing 4 ZigBee nodes has a $5 \mathrm{~dB}$ variability at different angles along the azimuth. They proposed an algorithm to obtain a single calibrated RSSI of the mobile system by averaging the individual RSSIs from the 4 nodes. In this way, the authors reduced the RSSI variability to $2 \mathrm{~dB}$.

Instead of doing mere averaging, and since smartphones can only be considered as a single node mobile system, our technique is orientation-aware and is able to correct the effect of orientation in a single transmitter-receiver system using commercial off-the-shelf smartphones, for an RF ranging system.

The rest of the paper is organized as follows. In Section II, we present our methodology to experimentally collect RSSI values using commodity hardware and open source software. In Section III, we present the results obtained when the methodology is applied to a commercial smartphone, and we analyze the sensitivity of the smartphone RSSI measurements to various parameters and correction techniques. We conclude this paper in Section IV with some conclusions.

\section{Methodology}

In the following, we present our methodology to perform wireless experiments for LTE, and we extend some of our 
experiments to Bluetooth in order to show that our findings expand beyond LTE.

\section{A. Controlled experimental setup}

In this section, we present our controlled experimental setup for LTE and Bluetooth experimentation based on commodity hardware and on open-source software [22].

- LTE signal generation. Instead of using specialized hardware for the generation of the LTE cellular network signals, we use OpenAirInterface (OAI) [23], a software implementation of an LTE cellular network that can run on general-purpose processors. The Core Network (CN) and the Radio Access Network (RAN) components of OAI usually run on two different machines to ensure real-time performance. As there is no need for Mobile data in our experiments, deactivating it allows us to reduce the computing load on the processor. Hence, both the CN and RAN components can run on the same machine. We use an HP Zbook laptop running Ubuntu 16.04 LTS with Intel i7-6thgen processor and $32 \mathrm{~GB}$ of RAM. We connect the laptop to an Ettus B210 [24] Universal Software Radio Peripheral (USRP). We use a band 7 duplexer to connect both the Rx and Tx channels of the USRP to an ETS-Lindgren's 3115 double-ridged horn $\mathrm{Rx} / \mathrm{Tx}$ antenna. This is a directional antenna with linear polarization (mono-polarized) having a gain of $10 \mathrm{~dB}$ at $2.5 \mathrm{GHz}$. In our setup, it is called the source or the transmitting antenna, as shown in Figure 1.

- Bluetooth signal generation. In order to generate the Bluetooth signals, we used two types of devices. We used an Arduino Bluetooth dongle model "Blend Micro" from Red Bear Labs [25]. It uses Bluetooth 4.0 Low Energy. We programmed the dongle to broadcast Bluetooth beacons and connected it through USB for powering. In addition to the Bluetooth dongle, we used a Fossil smartwatch [26] with Bluetooth Low Energy 4.2 and firmware version HW0.0.2.6r.v1 as a second source. This source is typical of what can be found from a real Bluetooth low energy consumer device. For the Bluetooth experiments inside the anechoic chamber, the Bluetooth dongle is directly mounted to replace the transmitting horn antenna. The dongle generates the Bluetooth signal and transmits it through its own embedded antennas.

- Device Under Test. For the device under test, we use a Nexus 5X smartphone running Android 7. To attach the smartphone to the network, we program a SIM card with the authentication parameters that we defined in the OAI database.

- Controlled environment with programmable robotic apparatus. We perform our experiment in an anechoic chamber that has programmable robotic equipment both at the transmission and reception sides. As shown in Figure 1, the reception platform is a two-axis positioning system that rotates along the two axes $x$ and $y$ : $\varphi$ (Azimuth, the angle between $x$ and $z$ axes) and $\theta$ (Roll, the angle between $y$ and $z$ axes). $y$ can rotate $180^{\circ}$ (from $-90^{\circ}$ to $+90^{\circ}$ ) whereas $x$ can make a $360^{\circ}$ rotation. The transmission platform can only rotate along the $x$-axis. In Figure 1, the transmission system is positioned at $\varphi=0^{\circ}$.
By combining the two-axis rotations, we can obtain measurements of the RSSI using the smartphone in different orientations. The reception and transmission are separated by 4 meters and connected to a controller system (Apparatus controller) placed outside of the chamber, which allows us to program the rotation of the platforms by defining the rotation range, the step, and the time duration it remains at each orientation.

\section{B. Outdoor experimental setup}

The controlled experimental setup allows us to perform reproducible experiments with a fine grain control of each experimental parameter. However, in reality, we might have transmission diversity (e.g., for LTE) and multipath transmission due to signal reflections (e.g., for both LTE and Bluetooth).

The goal of the outdoor experimental setup is to assess how the complexity observed outdoor impacts our findings.

- Cellular outdoor setup. In order to assess the accuracy of the RSSI measurement from a smartphone with a real LTE base station, we use the same LG Nexus 5x phone described in section II-A inside the main transmission lobe of an LTE base station, at a distance of 170 meters. The direction of the main lobe is obtained from the official maps provided by the French National Agency of Radio-frequencies (ANFR) [27]. We lock the smartphone on the same band we had used in the controlled environment, that is on band 7 .

We rotate the smartphone on the two axes $\varphi$ and $\theta$ in order to test for different relative orientations between the smartphone and the source. At each orientation, we collect at least 20 RSSI samples and compute the mean value.

- Bluetooth outdoor setup. We use a meeting room that contains tables and chairs in order to carry out the Bluetooth experiment in a realistic environment, i.e. in the presence of reflections (by outdoor setup, we mean outside of the anechoic chamber). We place the Bluetooth dongle in the direct line of sight with respect to the smartphone, at a distance of 4 meters, that is the same distance as between the source and the reception in the controlled environment.

We again rotate the smartphone on the two axes $\varphi$ and $\theta$ to test for different relative orientations between the smartphone and the source. At each orientation, we wait for 1 minute (roughly 5 RSSI samples) and compute the mean value.

\section{Logging the measurements}

We log the measurements onto the apparatus controller (LOGS-1) and onto the smartphone (LOGS-2). The logs are timestamped with the time from the local clock, as shown in Figure 1. We synchronize the timestamps in a post-processing phase.

For the smartphone log collections, we use the Electrosmart mobile application [9] in order to collect the $\mathrm{Rx}$ power (RSSI) and the device orientation from the IMU sensors.

The apparatus controller creates timestamped logs of the values of its rotation axes each time it reaches a pro- 


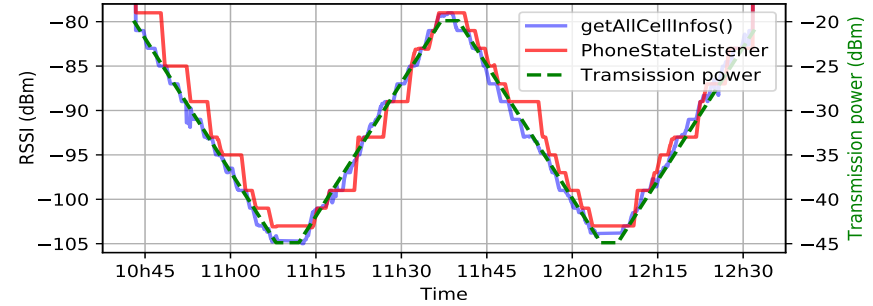

Fig. 2. Comparing Android API to get the LTE RSSI while varying the Tx power (right y-axis). The getAllCellInfos method (blue line) is more reliable and more sensitive to changes in the signal strength than the PhoneStateListener method (red line).

grammed orientation. The values are expressed in terms of $\varphi$ and $\theta$ in degrees. These logs are used as ground true values for device orientation inside the chamber.

\section{C.1 Getting the LTE RSSI on Android}

The Android Application Programming Interfaces (APIs) offer two possibilities to get the LTE RSSI. The two methods are the following.

- PhoneStateListener is a callback-based method. It works by registering a listener to monitor the changes in the network signal strength, and get a callback whenever the signal strength changes [28].

- getAllCellInfo () is an explicit call to the operating system by invoking the getAllCellinfo() method to fetch the most recent signal strength measured by the hardware [29].

The two methods are supposed to report the changes in the signal strength of the network. However, which one is better to monitor the changes in the signal strength? In order to compare between the two methods, we place an LG Nexus 5X smartphone in the anechoic chamber as shown in Figure 1. Subsequently, we vary the transmission between $-45 \mathrm{dBm}$ and $-20 \mathrm{dBm}$ in $1 \mathrm{~dB}$ steps, one step per minute. We record the RSSI on the smartphone using the two aforementioned methods. We trigger a call to getAllCellInfo() every 1 second. The results are shown in Figure 2. The method getAllCellInfos() is more sensitive to the changes in the RSSI than the PhoneStateListener method. For example, at time 10h45, PhoneStateListener keeps giving the same RSSI $(-80 \mathrm{dBm})$ regardless of the fact that the transmission (Tx) power has dropped from $-20 \mathrm{dBm}$ to $-25 \mathrm{dBm}$, then it suddenly updates to $-85 \mathrm{dBm}$. In contrast, getAllCellinfos() follows exactly every update in the Tx power.

For the rest of this work, we choose the getAllCellinfos() method in order to measure the RSSI on the smartphone.

\section{C.2 Getting the Bluetooth RSSI on Android}

The Bluetooth RSSI on Android is obtained by registering a broadcast receiver that listens to events (called intent in Android) triggered by the Bluetooth Adapter on the smartphone. Each time a new Bluetooth source is detected, a BluetoothDevice.ACTION_FOUND intent is received. We extract the RSSI value from an extra field in this intent called BluetoothDevice.EXTRA_RSSI.

\section{C.3 Getting the smartphone orientation on Android}

Android APIs give access to the smartphone orientation using the Rotation Vector Sensor (RVS). RVS is a software sensor that combines many hardware sensors readings (Accelerometer, Magnetometer, and Gyroscope) to estimate the device's orientation in space. The RVS returns a vector that can be transformed into a quaternion of orientation. Quaternions [30] are 4 dimensional complex vectors. They can be averaged by slerping [30] (Spherical Linear intERPolation) and, in contrast to Euler angles, they do not suffer from Gimbal lock, which is a loss of a degree of freedom when representing the orientations in a $3 \mathrm{D}$ space [31].

\section{Experimental limitations}

For measurement acquisition, we faced some limitations. First, the refresh rate of the smartphone signal strength is in the order of 1 second at the best. This is due to power optimizations restricting the number of messages exchanged between the device's baseband (which has a higher refresh rate) and the Android OS. A higher refresh rate would shorten the time spent collecting the calibration data.

Second, the two-positioner system can only rotate along two axes, which means we cannot test all the relative orientations of the device with respect to the source. This can be solved by rotating the source itself along $\varphi$. We limit our study to a subset of relative orientations of the smartphone with respect to the source by considering two polarizations of the source (horizontal and vertical polarizations). All the details about the calibration process we are presenting in this work can be replicated for any different orientations or polarizations without loss of generality.

Last, the LTE RSSI values range from $-113 \mathrm{dBm}$ to $-51 \mathrm{dBm}$ [32]. Since RSSI values are capped at $-51 \mathrm{dBm}$, we made sure in all our experiments that measurements had never been capped.

\section{EXPERIMENTAL EVALUATION OF THE ACCURACY OF THE RSSI MEASUREMENTS FROM A SMARTPHONE}

The reception performance of a smartphone can be affected by different parameters such as the device orientation, the source position (the source pointing with its main radiation lobe towards the device), the source orientation (the source no longer pointing with its main radiation lobe towards the device), and the source transmission (Tx) power.

$$
\bar{P}=P_{r}+\alpha P_{r}+\beta P_{r}+\gamma P_{r}+\rho P_{r}
$$

The effect of these parameters can be corrected using correction factors in order to obtain a correct RSSI. Equation (1) shows $\bar{P}$ the corrected RSSI where $P_{r}$ is the raw RSSI measured from the smartphone, and $\alpha, \beta, \gamma, \rho$ are the correction factors to compensate for the orientation, the source position, the source orientation, and for the source Tx power, respectively.

In this section, we start by measuring the reference RSSI that will be a ground true target for the corrected RSSI $\bar{P}$. 
Subsequently, we explore the sensitivity of the measured RSSI from a smartphone with respect to the device orientation, the source position, the source orientation, and to the source Tx power in a controlled environment. In each scenario, we propose a correction technique to estimate $\bar{P}$. Finally, we evaluate the characteristics of an outdoor environment on the measured RSSI.

Most of our experiments are performed on LTE, but we have also performed some experiments on Bluetooth in order to show how our findings in LTE can be extended to other wireless technologies.

\section{A. Measuring the reference RSSI}

We measure the actual LTE RSSI at the reception point using a spectrum analyzer [33]. We use a horizontal polarization at the source. On the spectrum analyzer we place a horn antenna identical to the transmitting antenna with the same polarization as the source. By removing the antenna gain $(10 \mathrm{~dB})$ and compensating for cable loss $(1 \mathrm{~dB})$, the RSSI measured at the reception is $\mathbf{- 5 4} \mathbf{~} \mathbf{B B m}$. We call this RSSI the reference RSSI.

\section{B. Evaluating the effect of the device orientation}

In this section, we evaluate the accuracy of the smartphone's raw LTE RSSI inside an anechoic chamber for a mono-polarized antenna and quantify the effect of the smartphone orientation with respect to the source on the RSSI.

In order to compare between the smartphone measurements and the reference RSSI, we replace the horn antenna at reception with a smartphone. In order to study the effect of the smartphone orientation on the RSSI, we place the device in 684 different orientations along two axis, $\varphi$ and $\theta$, in front of the transmitting antenna, as illustrated in Figure 1. At each position, we collect the RSSI as well as the device orientation.

We keep the device at each orientation for 10 seconds. Then, we average the RSSI and the quaternions measured for each orientation: RSSIs are averaged in Watt and the results are converted into $\mathrm{dBm}$; the orientation is obtained by slerping the quaternions in order to obtain a representative quaternion for each orientation. To verify the stability of the RSSI at each orientation during the measurement period (10 seconds), we compute the standard deviation of the RSSI over time for each orientation. The mean standard deviation of the RSSIs for all the 684 orientations is only $0.06 \mathrm{dBm}$.

To verify the reproducibility of the measurements, we repeated the same experiment 10 times. For every experiment, we started the experimental process from scratch: we set up the LTE network, we calibrate the orientation sensors of the smartphone [34], we position it on the twopositioner system, next we launch the controller program to start rotating the device and to collect the measurements. For all 10 experiments, the mean standard deviation of the RSSI for each orientation is $0.51 \mathrm{dBm}$ and $5.5^{\circ}$ mean angle error. Finally, we merge all 10 experiments together and, for each orientation, we compute the average
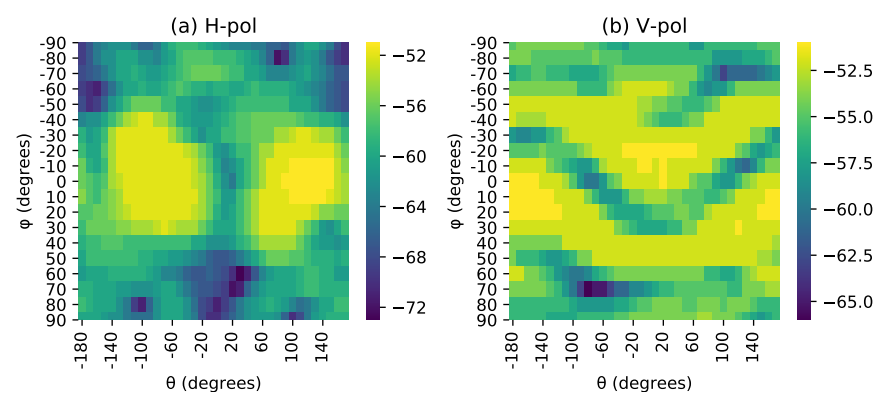

Fig. 3. Heatmap of the LTE RSSI (in $\mathrm{dBm}$ ) obtained for the LG Nexus 5X for 684 orientations made for horizontal and vertical polarization of the source. The reception performance is very sensitive to the device orientation.

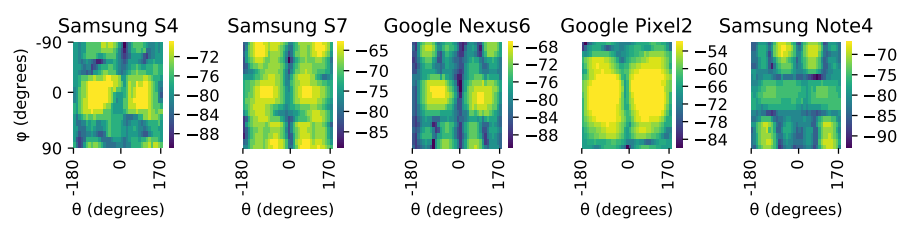

Fig. 4. Heatmap of the LTE RSSI (in dBm) for 5 different smartphones evaluated using our methodology. The reception performance is sensitive to the device orientation for all smartphones, but the characteristics of the sensitivity is very different from a smartphone to another.

RSSI and the mean quaternion. For the rest of our study, we use the resulting averaged RSSIs and orientations. The vertical polarization was also evaluated by repeating the same measurement procedure as described for the horizontal polarization.

By placing the smartphone in 684 different orientations with horizontal polarization, and by measuring the RSSI, we obtain a heatmap of the RSSI shown in Figure 3(a). We can see a large variability of the RSSI across the different orientations. The optimal RSSI we measured was $-51 \mathrm{dBm}$ at $\theta=+90^{\circ}$ and $\varphi=0^{\circ}$ (same orientation as depicted in Figure 1), which is $3 \mathrm{~dB}$ more than the reference RSSI $(-54 \mathrm{dBm})$. At some orientations, the reception performance is very poor with a minimum of $-73 \mathrm{dBm}$. The RSSIs for the same 684 orientations, repeated for the vertical polarization are shown in Figure 3(b). At this polarization, the RSSI also exhibits a large variability with an offset of $15 \mathrm{~dB}$ between the maximum and the minimum RSSI.

In addition to the Nexus $5 \mathrm{X}$, and using the same methodology, we evaluated the reception performance of 5 different smartphones. We tested the Samsung S4, Samsung S7, Samsung Note 4, Google Nexus 6, and Google Pixel 2. We observe in Figure 4 that all smartphones show their measured LTE RSSIs to be largely impacted by the device orientation. However, the reception patterns are very different from one device to another. This difference can be due to many factors, such as the dimensions of the smartphone, the casing, the number of antennas used, and their location within the smartphone.

We also expect to have specific orientations where the RSSI is optimal, this will happen when the smartphone antennas are aligned with the polarization of the source. 


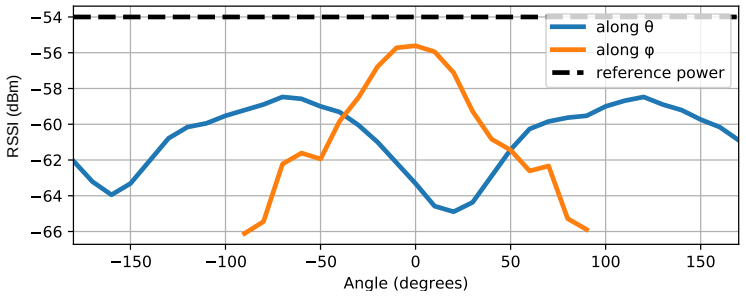

Fig. 5. Mean LTE RSSI along $\varphi$ and $\theta$. The RSSI is optimal when the antenna is in co-polarization with the source $\left(\theta=120^{\circ}\right.$ and $\theta=$ $\left.-60^{\circ}\right)$ and when the smartphone is oriented towards the source $(\varphi=$ $\left.0^{\circ}\right)$

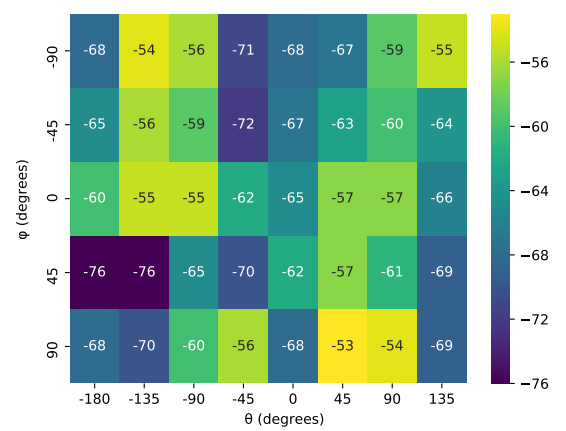

Fig. 6. Heatmap of the Bluetooth RSSI (in $\mathrm{dBm}$ ) obtained for the LG Nexus 5X for 40 orientations in a controlled environment. The reception performance is also sensitive to the device orientation for Bluetooth.

In antenna theory, Polarization Matching [35] (or copolarization) means that the receiver and the transmitter have the same polarization, thus the power loss being minimal. In contrast, cross-polarization yields minimal power. So by monitoring the RSSI and by knowing the polarization of the source, we can determine at which orientation the co-polarization happens.

In order to determine the polarization scheme of the smartphone, we plot the mean RSSI along the two axes of rotation as shown in Figure 5. Along $\varphi$ axis, the maximum power is received when the smartphone is in the main transmission lobe $\left(\varphi=0^{\circ}\right)$. We also see that the maximum power along $\theta$ is produced at angles $+120^{\circ}$ and $-60^{\circ}$, and lowest reception occurs when the smartphone is rotated by $90^{\circ}$ along $\theta$. Hence, smartphone antennas are affected by their relative orientation with respect to the source and the optimal performance is observed when their polarization matches the polarization of the source.

For the rest of this work, and for the sake of simplicity, we assume that the source polarization does not change and that it is known beforehand (in practice, we only consider the horizontal polarization). This might not be true in practice, but we can deduce the polarization of the source using the property of polarization matching, that is by placing the device in different polarizations, by monitoring orientations with optimal RSSI, and by knowing the characteristics of the reception pattern of the smartphone. This procedure to find the source polarization can be performed using our mobile application prototype [13].

Finally, in order to validate that our results expand be- yond LTE, we explored the effect of the device orientation for Bluetooth RSSI measurements. We used the Arduino dongle as the source in our experimental setup, and we rotated the smartphone using the two-axis positioning system. In Figure 6 we show the RSSI measured for the 40 different orientations which have been measured. We can see that the measured Bluetooth RSSI is also very sensitive to the device orientation with up to $23 \mathrm{~dB}$ difference between the minimum $(-76 \mathrm{dBm})$ and maximum $(-53 \mathrm{dBm})$ measured RSSI. This proves that orientation does also affect the RSSI accuracy for the Bluetooth power measurements.

In summary, the measured RSSI from COTS smartphones is sensitive to the device orientation. This sensitivity holds for multiple devices and for different wireless technologies.

\section{Correcting the effect of the device orientation}

In this section, we show that it is possible to correct the effect of the device orientation on the measured LTE RSSI. We use the orientation sensors of the smartphone to build calibration matrices, which are used to compute a correcting factor which is subsequently applied to the measured RSSI for each orientation

Let $Q$ be a matrix of orientation Quaternions, and let $P$ be a matrix of RSSI having the same dimensions as $Q$. We call them calibration matrices. In order to build these matrices, we rotate the smartphone as shown in Figure 1, and for each orientation, we fill up the matrix $Q$ with the measured quaternion, and the matrix $P$ with the offset between the raw measured RSSI at a given orientation and the reference RSSI we measured in Section III-A. Therefore, in the element with coordinates $(i, j)$ in matrix $Q$ we have an orientation, and in the element with the same coordinate in matrix $P$ we have the offset to be applied to the measured RSSI for the specific orientation stored in matrix $Q$. Once we have these two matrices, whenever we place a device in the orientation defined by a given quaternion $q_{i}$, we compare it against all the quaternions in $Q$ and we compute the relative angle. The closest quaternion in $Q$ is the one with minimal angle to $q_{i}$. We use its coordinates in $Q$ to obtain the corresponding correction offset from $P$ and apply it to the raw measured RSSI to obtain the corrected RSSI.

To validate this correction technique, we built the calibration matrices by placing the device at $\theta=-180^{\circ}$ and $\varphi=-90^{\circ}$. Subsequently, we varied $\theta$ from $-180^{\circ}$ to $+170^{\circ}$ in $10^{\circ}$ steps. We did that for every $\varphi$ ranging from $-90^{\circ}$ to $+90^{\circ}$ in $10^{\circ}$ steps. At each step, we collected the RSSI and the rotation quaternion. Next, we inserted them into the matrices with the corresponding $\varphi$ and $\theta$ coordinates. The dimensions of the matrices $P$ and $Q$ is $36 \times 19^{1}$.

As a next step, we selected 100 random orientations for the device under test by computing 100 random couples $(\varphi$,

1 We computed calibration matrices for 5 additional devices: a Samsung S4 and S7, a Nexus 6, a Pixel 2, and a Note 4. We do not discuss the details of these other devices in the rest of this paper, since they provide us with similar conclusions, however we make all these matrices publicly available [13] so that the interested reader can easily reproduce our results for different devices. 


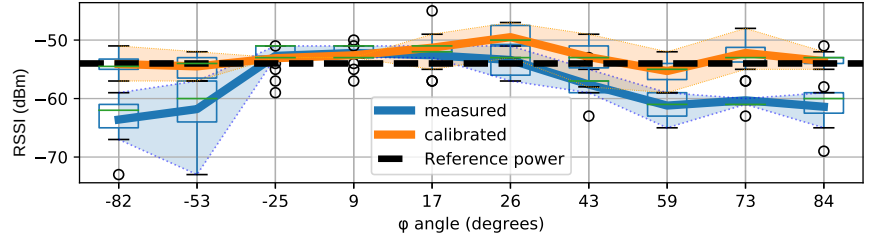

Fig. 7. RSSI correction results for the 100 random orientations along $\theta$ and $\varphi$. The colored area represents the variability of the measurements along $\theta$. The corrected RSSI (orange) is closer to the reference $R S S I$ (black dashed line), and less variable $(2.4 \mathrm{dBm}$ RMSE) as compared with the raw measured signal (shown in blue, with 6.4 dBm RMSE).

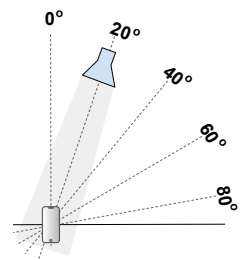

(a)

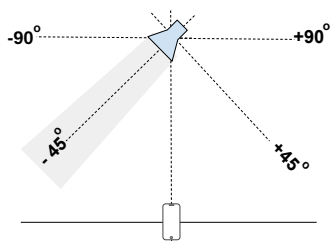

(b)
Fig. 8. (a) Changing the source's position with respect to the smartphone. (b) Pointing the source's main lobe at different angles with respect to the smartphone.

$\theta)$ selecting 10 random values for $\varphi$ within the range $\left[-90^{\circ}\right.$, $\left.+90^{\circ}\right]$ and 10 random values for $\theta$ within the range $\left[-180^{\circ}\right.$, $\left.+170^{\circ}\right]$ that were not in $Q$. Our goal is to obtain random coordinates which do not have an exact match in Q. The source polarization and Tx power are kept unchanged.

In order to correct the measured RSSI at a random orientation, we compare a given quaternion against all quaternions in $Q$ to get the closest quaternion and its corresponding coordinates in $Q$. We use these coordinates to get the RSSI offset in $P$ which allows us to correct the measured RSSI. Figure 7 shows that the corrected RSSI (orange line) is closer to the reference RSSI (dashed black line) and has a lower variability than the raw RSSI (blue line). In particular, the raw RSSI RMSE is $6.4 \mathrm{dBm}$, whereas the corrected received RSSI RMSE drops to $2.4 \mathrm{dBm}$.

In summary, our proposed technique for correction of the effect of the device orientation on the LTE measured RSSI significantly improves the measurements accuracy. The RMSE error is reduced by $4 \mathrm{~dB}$.

\section{Evaluating and correcting the effect of the source posi- tion}

In this section, we consider the case where the source's location with respect to the smartphone is unknown, but the distance to the source is kept constant and the smartphone remains in the main transmission lobe of the source, see Figure 8(a).

To evaluate the impact of the source position on the measured RSSI, we rotate the source at $20^{\circ}, 40^{\circ}, 60^{\circ}$, and $80^{\circ}$ from the original orientation along the azimuth $(\varphi)$. We make sure that the source is shifted by the correct angle and that the smartphone is kept within the main transmission lobe by means of a laser beam. At each new

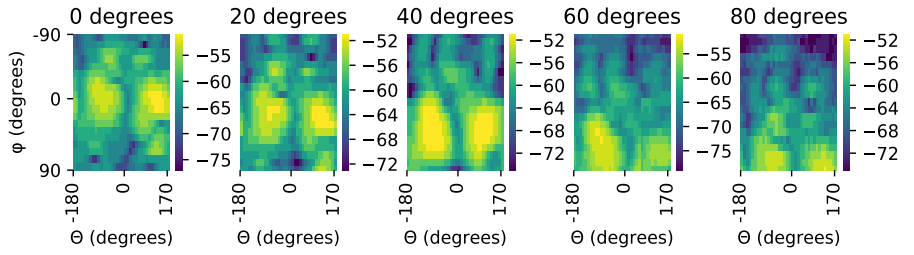

Fig. 9. Heatmap of the LTE RSSI of the Nexus $5 \mathrm{X}$ when the source is placed at different angles. The reception patterns are a shifted versions of the pattern at $0^{\circ}$ with the corresponding angle change in source position.

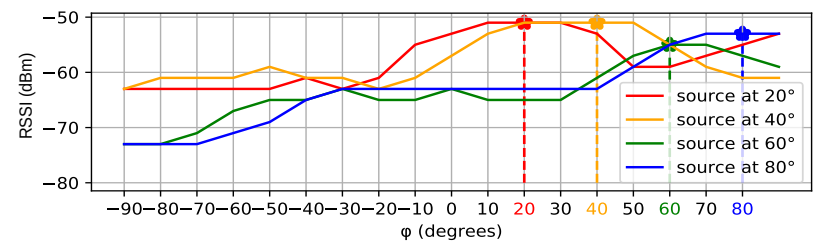

Fig. 10. Sweeping along the $\varphi$ axis to locate the source. The source position is found when maximum RSSI is measured

position, we measure the reference RSSI using a spectrum analyzer.

Next, we test whether we can reuse the calibration matrices obtained in Section III-C to correct the RSSI. Figure 9 shows the RSSI patterns for all the tested angles. We see that the patterns seem to be a shifted version of the reception pattern at $0^{\circ}$. Hence, the reception pattern should be preserved regardless of the source position with respect to the smartphone. To validate this hypothesis, we test the impact of shifting the calibration matrices we had collected when the source was at $\varphi=0^{\circ}$ in order to correct the measured RSSI at any angle. In order to compute the matrix shifting we have three steps: i) locating the new position of the source, ii) defining the angle shift from the $\varphi=0$ position to the new source position, iii) translating the calibration matrix of orientations $Q$ to adapt it to the new source.

In order to locate the source, we use the property of polarization matching we have described in Section III-B, where the reception is maximum when both the transmitting and the receiving antennas are aligned and copolarized. Our method is as follows. First, we assume that the source polarization is known, and we place the smartphone in the same polarization. Second, as illustrated in Figure 5 , and since the smartphone receives more power when $\theta$ is $+120^{\circ}$ or $-60^{\circ}$, so we place the smartphone at $\theta=+120^{\circ}$. Finally, we rotate the phone along the $\varphi$ axis from $-90^{\circ}$ to $+90^{\circ}$ and collect the RSSI for each value of $\varphi$. The source's position is determined when we measure the maximum RSSI at an angle $\varphi=\varphi_{m}$. We can see in Figure 10 that the RSSI increases gradually as we point the smartphone closer to the new source location. The maximum RSSI is received when the smartphone is directly aligned with the source along the azimuth $(\varphi)$, for instance, the red curve corresponding to the scenario with a source rotation of $20^{\circ}$ has its maximum when $\varphi$ is at $20^{\circ}$. This procedure to locate the source can be performed 


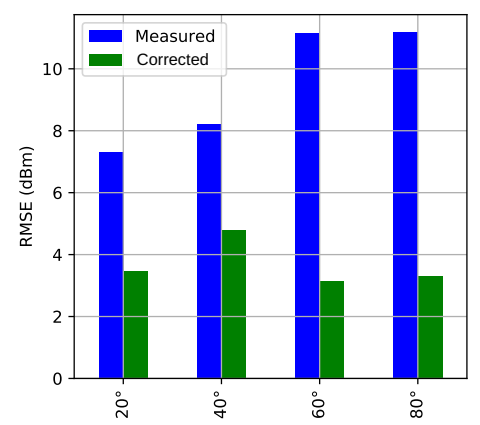

Fig. 11. RMSE for different source positions between the reference RSSI and the raw RSSI measurements (in blue), and between the reference RSSI and the corrected RSSI measurements (in green). The impact of the source position on the measured LTE RSSI can be corrected.

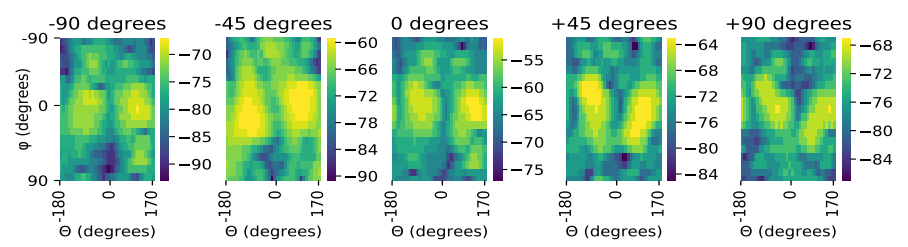

Fig. 12. Heatmap of the LTE RSSI of the Nexus 5X for different orientations of the main lobe. The reception patterns are impacted by the orientation of the source main lobe.

using our prototype mobile application for calibration [13].

Once we have located the source, we need to transform and shift the matrix of orientation quaternions $Q$ in order to adapt it to the new source position. We apply quaternion rotation using the relative quaternion describing the rotation from $\varphi=0^{\circ}$ to $\varphi=\varphi_{m}$, that is the new source position in the azimuth. Once $Q$ is transformed, we can apply the correction to the measured RSSI using the technique previously presented in Section III-C.

Figure 11 shows the RMSE as compared to the reference RSSI for the raw measurements (in blue) and for the corrected ones (in green). We can see that a modification of the source position can significantly increase the RMSE (up to $11.1 \mathrm{dBm}$ at $80^{\circ}$ ). However, we see a significant improvement of our correction technique when the source position is unknown with an RMSE between $3.3 \mathrm{dBm}$ and $4.8 \mathrm{dBm}$, so an average reduction in RMSE of $5.7 \mathrm{~dB}$.

In summary, when the smartphone is within the main transmission lobe of the source, but the source position is unknown, the measured LTE RSSI is a shifted version of the measured RSSI when the source is in front of the device. However, we have presented a correction technique that allows us to correct a large fraction of the measured LTE RSSI error, with an average reduction in RMSE of $5.7 \mathrm{~dB}$.

\section{E. Evaluating and correcting the effect of the source ori- entation}

Now, we consider the case where the source is no longer within the main transmission lobe. In Figure 8(b) it can

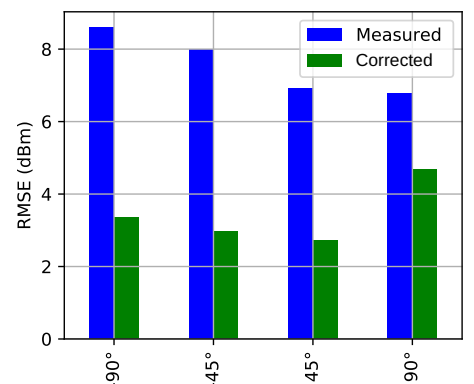

Fig. 13. RMSE for different source orientation of the main lobe between the reference RSSI and the raw RSSI measurements (in blue), and the reference RSSI and the corrected RSSI measurements (in green). The impact of the source orientation on the measured LTE $R S S I$ can be accounted for and corrected.

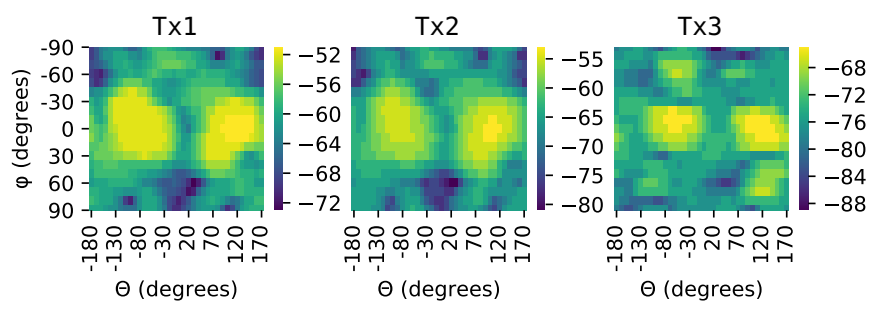

Fig. 14. The measured RSSI by the Nexus $5 \mathrm{X}$ for different Tx power levels. The reception patterns are impacted by the source Tx power.

be seen that the main lobe is pointed at different angles with respect to the smartphone. At each angle, we rotate the smartphone along $\varphi$ and $\theta$ in order to collect the RSSI. Figure 12 shows the heatmap of the RSSI in all tested cases. Unsurprisingly, we see that the measured RSSI is sensitive to the main lobe orientation.

We try to correct the RSSI when the source's main lobe is not directed towards the smartphone using the calibration matrices $P$ and $Q$ computed in Section III-C, when the smartphone is in the source main lobe, without any modification.

Figure 13 shows the RMSE between the raw measurements and the reference RSSI (in blue), and between the corrected measurement and the reference RSSI (in green). The rotation of the source's main lobe significantly increases the RMSE, which ranges between $6.8 \mathrm{dBm}$ and $8.6 \mathrm{dBm}$. Our correction technique, consisting in applying the calibration matrices without any modification successfully reduces the RMSE, now ranging between $3 \mathrm{dBm}$ and $4.7 \mathrm{dBm}$, i.e. an average reduction in RMSE of $4.1 \mathrm{~dB}$.

In summary, the orientation of the source's main lobe has a significant impact of the measured LTE RSSI. The proposed correction technique, consisting in applying the unmodified calibration matrices, can reduce the RMSE on average by $4.1 \mathrm{~dB}$. 


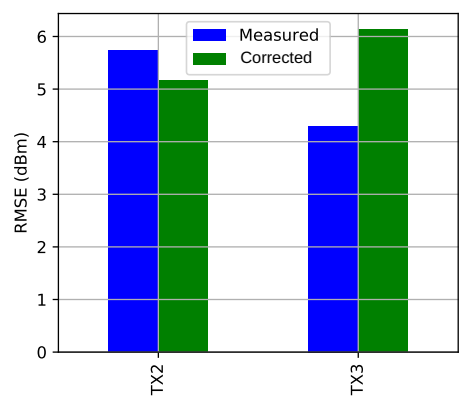

Fig. 15. Root Mean Squared Error (RMSE) for different source Tx power between the reference RSSI and the raw RSSI measurements (in blue), and the reference RSSI and the corrected RSSI measurements (in green). The impact of the source Tx on the measured LTE RSSI cannot be corrected with the mere application of the calibration matrices.

\section{F. Evaluating and correcting the effect of the source Tx power}

In this section, we consider a change in the source transmitted (Tx) power ${ }^{2}$. The position of the source and its polarization are the same as in Figure 1. We consider three different transmission power levels: Tx1 is the transmission power used in all the previous experiments, Tx2 is Tx1 reduced by $10 \mathrm{~dB}, \mathrm{Tx} 3$ is Tx1 reduced by $20 \mathrm{~dB}$. In order to achieve this reduction in transmitted power, we change the transmission attenuation parameter att_tx found in the configuration file of OAI. For each Tx power, we measure the reference RSSI at the receiver as explained in Section III-A. We measure $-54 \mathrm{dBm},-64 \mathrm{dBm}$, and $-74 \mathrm{dBm}$ for Tx1, Tx2, and Tx3, respectively. For each Tx power, we collect the RSSI measurements using the same procedure as described in Section III-C, i.e. by rotating the smartphone along $\theta$ and $\varphi$. Figure 14 shows the received patterns for the three different $\mathrm{Tx}$ power levels defined before. Unsurprisingly again, we see that the RSSI measurements are impacted by the Tx power.

Similarly to what we did in Section III-E, we try to correct the RSSI with the calibration matrices $P$ and $Q$ previously computed in Section III-C, when the source is emitting a Tx power level TX1, without any modification.

Surprisingly enough, this correction technique fails to improve the mean RMSE. Indeed, Figure 15 shows that for the corrected measurements the RMSE corresponding to Tx2 is slightly decreased, but it is increased for Tx3. The average RMSE for the corrected measurements is increased by $0.6 \mathrm{~dB}$.

The reason for this effect is that changing the Tx power affects the reception patterns of the smartphone. Indeed, in LTE, smartphones work with a Multiple Input Multiple Output (MIMO) technology. Depending on the network quality, the smartphone can select a single antenna or it can combine the different antennas to optimize the received signal power [36]. This is called antenna diversity. There

\footnotetext{
2 Note that we can extend the results of this section to a modification of the distance to the source, as increasing the distance to the source is equivalent to reducing the transmitted power.
}
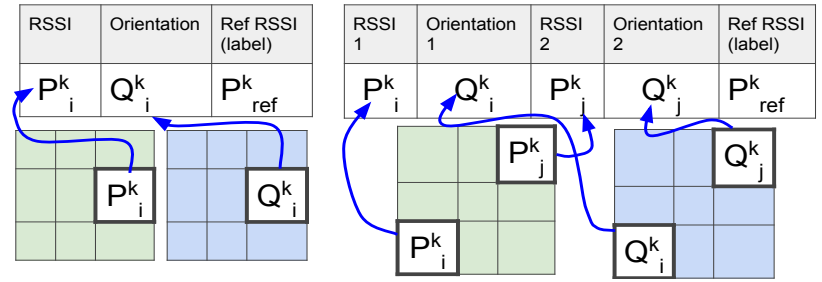

Fig. 16. ML models for RSSI corrections. One-couple (left) and Two-couple models (right). The model maps the raw RSSI in each orientation to the reference RSSI corresponding $\mathrm{k}^{\text {th }} \mathrm{Tx}$ power.

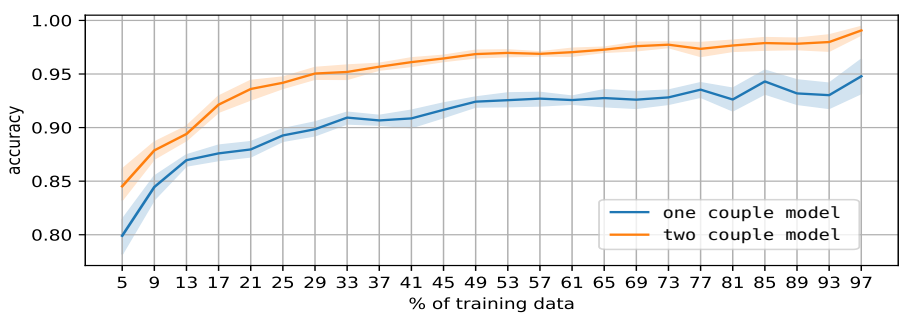

Fig. 17. Random Forest accuracy for selection of the right RSSI for different amounts of training data. The average (solid line) over 10 independent trainings is computed together with its uncertainty interval for a $95 \%$ level of confidence (colored area). Even a moderate amount of training is enough to have a good accuracy.

exist different techniques for diversity combining [37]. The smartphone may use only one of the antennas for reception (switched diversity) or it can combine the incoming signal from all antennas according to their respective Signalto-Noise Ratio (SNR), a technique called Maximum Ratio Combining (MRC). This antenna diversity significantly complicates the correction process since there is no previous knowledge about which calibration matrix should be used for the measured RSSI.

In order to deal with this issue, we evaluate the performance of two machine learning models based on a Random Forest algorithm to predict the correct RSSI. The first one uses as features one couple (RSSI, device orientation) at a time selected from one of the reception pattern matrices presented in Figure 14 and labelled with the reference RSSI for this matrix. The second one uses as feature two (RSSI, device orientation) couples at a time selected independently from the same calibration matrix labelled with the known reference RSSI for this matrix. The machine learning model building process is illustrated in Figure 16. The illustration shows how in the one-couple model, one point at a time is taken from the reception patterns $(P)$ and orientation $(Q)$ matrices, then labelled with the correct reference RSSI $\left(P_{\text {ref }}^{\mathrm{k}}\right)$. Whereas, in the two-couple model, two points at a time are taken from both matrices, and labelled with the reference RSSI corresponding to the given Tx power. The two-couple model captures more features of the smartphone's reception pattern, which allows the model to distinguish with higher accuracy which transmission power the received pattern corresponds to. After training the models, we take one test orientation (in case of the one-couple model) or two test orientations (in case of the two-couple model). 
In order to perform the evaluation, we train the model with $X \%$ of the couples (or pairs of couples), $\mathrm{X}$ ranging from $5 \%$ to $97 \%$ in $4 \%$ steps. The remaining data is used for cross-validation. For each X, we repeat the training 10 times with another uniformly distributed random subset of $\mathrm{X} \%$ of couples (or pairs of couples).

The accuracy of the model to give the correct RSSI is shown in Figure 17. It can be see that with $30 \%$ of training data, the model can predict with $90 \%$ accuracy the reference RSSI with the one-couple model (blue curve). The accuracy increases to $95 \%$ if we use the two-couple model (orange curve). Even with 5\% of training data, the accuracy is $80 \%$ for the one-couple model. This means that a simple Random Forest model can capture the reference RSSI from a single measurement with high accuracy even with a small amount of training data.

In Summary, we have shown that the source transmission power has a significant impact on the accuracy of LTE RSSI measurement with a RMSE up to $5.7 \mathrm{dBm}$ due to antenna diversity optimizations on the smartphone. However, we can dramatically improve the accuracy by using machine learning with a simple Random Forest model and with minimal training. We have shown that a $90 \%$ accuracy can be achieved with $30 \%$ training and using a one-couple model.

\section{G. Evaluating the effect of an outdoor environment}

So far, we have evaluated the RSSI accuracy of a smartphone in a controlled environment with a mono-polarized emitting antenna. In this section, we evaluate the accuracy of the RSSI with a multi-polarized antenna in an outdoor environment with reflections and multipath. Moreover, the LTE base stations nowadays make use of transmission diversity, such as spatial diversity and polarization diversity where the signal is transmitted at two perpendicular polarizations from an antenna array in order to improve cellular reception. Polarization diversity provides a gain of up to $12 \mathrm{~dB}$ as compared to single polarization [38]. In Figure 18(a), we show a typical sector antenna's interior used at a base station. We can see the arrangement of multiple antennas with vertical and horizontal alignment in order to achieve polarization diversity and thus to minimize the polarization mismatch at reception.

In order to assess the accuracy of the RSSI measurement from a smartphone under these conditions, we follow the methodology already described in Section II-B, and we place the smartphone in 88 different orientations along the two axes $\varphi$ and $\theta$ (which are a subset of the 684 orientations we tested in the controlled environment) inside the main transmission lobe of an LTE base station, see Figure 18(b). We compute the variability of the measurements collected outdoors, and we compare it to the variability of the measurements collected in the controlled environment with a mono-polarized source, for the same set of orientations.

In Figure 18(b), we can see that the measurements collected outdoors are less variable than those we obtained in the controlled environment. The median variability is

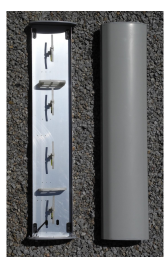

(a)

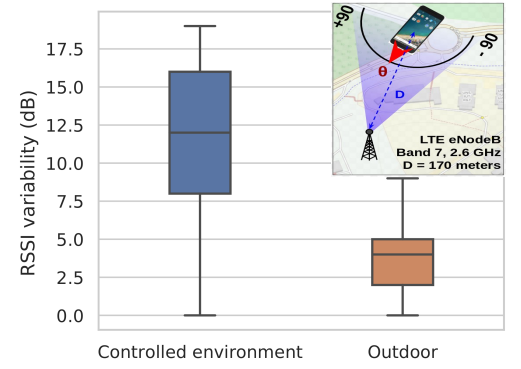

(b)
Fig. 18. (a) Interior of sector antenna (MIT Computer Science \& Artificial Intelligence Lab). (b) Outdoor evaluation results.

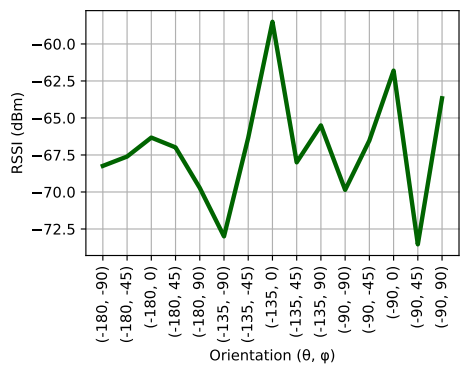

(a) Arduino Bluetooth source

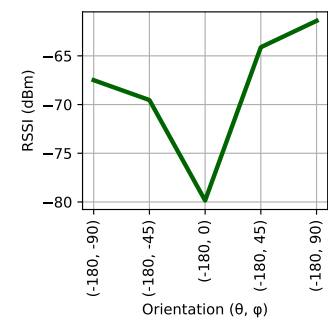

(b) Smartwatch source
Fig. 19. Sensitivity of the Bluetooth RSSI to the device orientation in an office environment. We observe a large variability of the RSSI with the device orientation for both sources.

about $4 \mathrm{~dB}$ outdoors, as compared to the $12 \mathrm{~dB}$ variability in the controlled environment. We perform two additional independent experiments with a different subset of orientations using the same experimental arrangement (omitted here due to the lack of space), and both confirm that the LTE RSSI outdoors is less variable and less affected by the orientation of the smartphone than the LTE RSSI in controlled environment.

Since the LTE base station transmits the same signal at two perpendicular polarizations (vertical and horizontal polarizations), the smartphone antennas can compensate for the errors introduced by the radiation patterns by means of a more efficient combination of signals coming from multiple antennas. Therefore, the polarization diversity used at transmission minimizes the chance of polarization mismatch (cross-polarization). Hence, the effect of orientation on the RSSI in an outdoor environment is minimized (low directivity).

In Section III-B we have seen that the Bluetooth RSSI measurements are also sensitive to the device orientation. However, Bluetooth does not use the technique of transmission diversity. For this reason, we have evaluated the impact of the device orientation in an office environment (described in Section II-B) for two different types of sources: an Arduino dongle and a smartwatch.

Figure 19 shows the measured Bluetooth RSSI and its variability with the smartphone orientation. We can see that the orientation has a large impact on the measured Bluetooth RSSI in a realistic environment for both types 
of sources. Interestingly enough, we observe that the minimum measured RSSI is not obtained for the same orientation. This is due to a different polarization of the two sources.

In summary, in outdoor (uncontrolled) environments, transmission diversity succeeds to compensate most of the effect of the device orientation on the measured LTE RSSI. However, for Bluetooth, since it does not make use of transmission diversity, even in outdoor environments, the smartphone orientation has a large impact on the measured Bluetooth RSSI.

\section{Conclusions}

In this work, we have evaluated the performance of a Commercial Off-The-Shelf (COTS) smartphone for RSSI measurements. We have three main take-home messages. First, a COTS smartphone cannot be used out of the box to perform accurate RSSI measurements with a monopolarized source in a controlled environment since these measurements are highly sensitive to the smartphone orientation, source position, source orientation, and to the source transmission power. Second, we propose efficient correction techniques based on the IMU sensors embedded in the smartphone to correct the RSSI measurements for all the conditions which have been evaluated and described here. With these techniques, we can reduce the RSSI RMSE to less than $5 \mathrm{dBm}$. Third, in an outdoor (uncontrolled) environment, transmission diversity (a technique that can be found, for instance,in $4 \mathrm{G}$ base stations), succeeds to mitigate the problem of accuracy due to the smartphone orientation. However, for protocols, such as Bluetooth, that do not support transmission diversity, the RSSI measurements are still highly sensitive to the device orientation.

In this work, we have mainly focused on LTE RSSI measurements, but we also extended our results to Bluetooth. In particular, we showed that Bluetooth RSSI measurements are sensitive to the device orientation even in realistic environments. The correction techniques we developed and described here for LTE should be evaluated for its use with Bluetooth in future investigations.

With the recent development of proximity tracking to help reducing the propagation of the COVID-19 disease, the need to perform accurate Bluetooth RSSI measurements becomes even more important. We do believe that this work provides a ground basis to build more accurate proximity estimations based on RSSI measurements.

\section{ACKNowledgements}

This work has been supported by the French government, through the UCAJEDI Investments in the Future project managed by the National Research Agency (ANR) with the reference number ANR-15-IDEX-01.

\section{REFERENCES}

[1] OpenSignal, "Mobile Analytics and Insights | Opensignal," Jan 2020, [Online; accessed 31. Jan. 2020].

[2] Tutela, ,"Jan 2020, [Online; accessed 31. Jan. 2020].
[3] CORDIS project, "Final Report Summary - EMF-NET (Effects of the exposure to electromagnetic fields: from science to public health) | Report Summary | FP6 | CORDIS | European Commission," Jan 2020, [Online; accessed 31. Jan. 2020].

[4] International Agency for Research on Cancer, "The Interphone Study," Jan 2020, [Online; accessed 31. Jan. 2020].

[5] GERONIMO Project, "Generalised EMF Research using Novel Methods - an integrated approach: from research to risk assessment and support to risk management | GERONIMO Project | FP7 | CORDIS | European Commission," Jan 2020, [Online; accessed 31. Jan. 2020].

[6] Robert Baan, Yann Grosse, Béatrice Lauby-Secretan, Fatiha El Ghissassi, Véronique Bouvard, Lamia Benbrahim-Tallaa, Neela Guha, Farhad Islami, Laurent Galichet, and Kurt Straif, "Carcinogenicity of radiofrequency electromagnetic fields," The Lancet Oncology, vol. 12, no. 7, pp. $624-626,2011$.

[7] The COSMOS project, ," [Online; accessed 31. Jan. 2020].

[8] XMobiSense app, ," Jan 2020, [Online; accessed 31. Jan. 2020].

[9] ElectroSmart, ," Jan 2020, [Online; accessed 31. Jan. 2020].

[10] Yixin Wang, Qiang Ye, Jie Cheng, and Lei Wang, "Rssi-based bluetooth indoor localization," in 2015 11th International Conference on Mobile Ad-hoc and Sensor Networks (MSN). IEEE, 2015 , pp. $165-171$.

[11] Google Apple, "Exposure notification, bluetooth specification," Tech. Rep., April 2020, Preliminary work, V1.2.

[12] Claude Castelluccia, Nataliia Bielova, Antoine Boutet, Mathieu Cunche, Cédric Lauradoux, Daniel Le Métayer, and Vincent Roca, "ROBERT: ROBust and privacy-presERving proximity Tracing," working paper, May 2020.

[13] Y BOUSSAD, M Naoufal Mahfoudi, A Legout, F Ferrero, L Lizzi, and W Dabbous, "Calibration android application - prototype and resources, https : / / github . com/Yanis-Boussad / smartphone _ calibration"

[14] Tan Zhang, Ashish Patro, Ning Leng, and Suman Banerjee, "A wireless spectrum analyzer in your pocket," in Proceedings of the 16th International Workshop on Mobile Computing Systems and Applications. ACM, 2015, pp. 69-74.

[15] Ana Nika, Zengbin Zhang, Xia Zhou, Ben Y Zhao, and Haitao Zheng, "Towards commoditized real-time spectrum monitoring," in Proceedings of the 1st ACM workshop on Hot topics in wireless. ACM, 2014, pp. 25-30.

[16] Andreas Achtzehn, Janne Riihihjärvi, Irving Antonio Barriía Castillo, Marina Petrova, and Petri Mähönen, "Crowdrem: Harnessing the power of the mobile crowd for flexible wireless network monitoring," in Proceedings of the 16th International Workshop on Mobile Computing Systems and Applications. ACM, 2015, pp. 63-68.

[17] OpenMoko project, "Openmoko," 2019.

[18] OsmocomBB project, "Osmocombb," 2019.

[19] Narseo Vallina-Rodriguez, Andrius Auçinas, Mario Almeida, Yan Grunenberger, Konstantina Papagiannaki, and Jon Crowcroft, "Rilanalyzer: a comprehensive $3 \mathrm{~g}$ monitor on your phone," in Proceedings of the 2013 Internet Measurement Conference (IMC'13), 2013, pp. 257-264.

[20] Chenglong Li, Emmeric Tanghe, David Plets, Pieter Suanet, Jeroen Hoebeke, Eli De Poorter, and Wout Joseph, "ReLoc: Hybrid RSSI- and Phase-Based Relative UHF-RFID Tag Localization With COTS Devices," IEEE Trans. Instrum. Meas., vol. 69, no. 10, pp. 8613-8627, Apr 2020.

[21] Valter Pasku, Mario Luca Fravolini, and Antonio Moschitta, "Effects of antenna directivity on RF ranging when using space diversity techniques," Measurement, vol. 98, pp. 429-438, Feb 2017.

[22] Y BOUSSAD, M Naoufal Mahfoudi, A Legout, F Ferrero, L Lizzi, and W Dabbous, "Open-Source 4G Experimental Setup," in 2020 IEEE International Symposium on Antennas and Propagation and North American Radio Science Meeting, Montreal, Canada, July 2020, IEEE.

[23] Navid Nikaein, Raymond Knopp, Florian Kaltenberger, Lionel Gauthier, Christian Bonnet, Dominique Nussbaum, and Riadh Ghaddab, "Openairinterface: an open lte network in a pc," in Proceedings of the 20th annual international conference on Mobile computing and networking. ACM, 2014, pp. 305-308.

[24] Ettus, "Ettus usrp b210," 2019.

[25] RedBearLab, "Blend micro," Web page, [Online; accessed October 2020].

[26] "Fossil, hybrid smartwatch commuter, FTW1150," Web page, [Online; accessed October 2020]. 
[27] Cartoradio - ANFR,,"Jan 2020, [Online; accessed 3. Feb. 2020].

[28] Android Developers, "Phonestatelistener," 2019.

[29] Android Developers, "Telephonymanager," 2019.

[30] EB Dam, M Koch, and M Lillholm, "Quaternions interpolation and animation. datalogisk institut," Københavns Universitet, 1998.

[31] A Alaimo, V Artale, C Milazzo, and A Ricciardello, "Comparison between euler and quaternion parametrization in uav dynamics," in AIP Conference Proceedings. AIP, 2013, vol. 1558, pp. $1228-1231$.

[32] ETSI, "3gpp ts 27.007 version 8.5.0 release 8," ETSI TS.

[33] Rohde\&Schwarz, "Fsl3 spectrum analyzer," 2019.

[34] calibrateMag, "Calibrating magnetometer sensor," 2019.

[35] Constantine A Balanis, Antenna theory: analysis and design, John wiley \& sons, 2016.

[36] Carl B Dietrich, Kai Dietze, J Randall Nealy, and Warren L Stutzman, "Spatial, polarization, and pattern diversity for wireless handheld terminals," IEEE transactions on antennas and propagation, vol. 49, no. 9, pp. 1271-1281, 2001.

[37] Sukhdeep Kaur, Jaipreet Kaur, and Manjit Sandhu, "Antenna diversity techniques,"

[38] Kyōhei Fujimoto, Mobile antenna systems handbook, Artech House, 2001. 